\title{
Engaging new dimensions in nonlinear optical spectroscopy using auxiliary beams of light
}

\author{
Jack S. Ford, David S. Bradshaw and David L. Andrews* \\ School of Chemistry, University of East Anglia, Norwich Research Park, Norwich NR4 7TJ, U. K.
}

\begin{abstract}
By applying a sufficiently intense beam of off-resonant light, simultaneously with a conventional excitation source beam, the efficiencies of one- and two-photon absorption processes may be significantly modified. The nonlinear mechanism that is responsible, known as laser modified absorption, is fully described by a quantum electrodynamical analysis. The origin of the process, which involves stimulated forward Rayleigh-scattering of the auxiliary beam, relates to higher order terms which are secured by a time-dependent perturbation treatment. These terms, usually inconsequential when a single beam of light is present, become prominent under the secondary optical stimulus - even with levels of intensity that are moderate by today's standards. Distinctive kinds of behaviour may be observed for chromophores fixed in a static arrangement, or for solution- or gas-phase molecules whose response is tempered by a rotational average of orientations. In each case the results exhibit an interplay of factors involving the beam polarisations and the molecular electronic response. Special attention is given to interesting metastable states that are symmetry forbidden by one- or two-photon absorption. Such states may be accessible, and thus become populated, on input of the auxiliary beam. For example, in the one-photon absorption case, terms arise that are more usually associated with three-photon processes, corresponding to very different selection rules. Other kinds of metastable state also arise in the two-photon process, and measuring the effect of applying the stimulus beam to absorbances of such character adds a new dimension to the information content of the associated spectroscopy. Finally, based on these novel forms of optical nonlinearity, there may be new possibilities for quantum non-demolition measurements.
\end{abstract}

Keywords: nonlinear optics, photon absorption, Rayleigh scattering, off-resonant laser light, quantum electrodynamics, rotational average, beam polarization, quantum non-demolition

\section{INTRODUCTION}

Single-photon absorption of light usually occurs through individual photons resonantly interacting with individual molecules. Multiphoton absorption results from the combined action of two or more photons - their net energy being absorbed in molecules with suitably placed energy levels. It is generally thought that photons outside the wavelength range of direct molecular absorption, either through single- or multi-photon process, have no effect on absorption. A challenge to this principle arose following the recognition that within a cooperative process, two neighboring molecules might each absorb an off-resonant photon having equal but opposite shifts from the resonant frequency - an electrodynamic interaction between the two molecules remediating energy conservation at each location by virtual photon coupling. ${ }^{1}$ In contrast, the analyzed process that follows, without entailing intermolecular coupling, also provides for off-resonant light to modify the characteristics of absorption, in this case for both one-photon and twophoton absorption. Specifically, we show (in section 2) that absorption rates are potentially changed by an off-resonant laser beam of sufficient intensity, through a nonlinear coupling mechanism within each molecule individually. The origin of such mechanisms is traced to higher order terms in the standard perturbation treatment of absorption which, although normally insignificant, become markedly prominent under the described conditions. A mechanism of similar nature can operate in molecular fluorescence, as we have shown in other recent work. ${ }^{2-4}$ By using our novel mechanism, an interesting potential outcome is that electronic absorption transitions that are normally symmetry forbidden may become allowed; since different selection rules arise for our process compared to conventional absorption. 
In experimental systems, the optically active molecules (or chromophores) will often be present in solution. Therefore, to extend the theory, the role of the surrounding material is now analyzed. Here, the characteristics of the light-matter interaction are qualitatively different compared to isolated molecules, and the random orientation of the molecules must also be taken into account. Two main developments are brought into the latter analysis. Firstly, since all media are both refractive and dispersive, the optical interactions within each molecule should be described with reference to the electronic influence of the surroundings upon the optical fields. In particular, the electric displacement induced by photons propagating through a solution is affected by the refractive index. Accordingly, the optical density of the medium (a bulk property) is introduced into the framework of the analysis that follows. Secondly, by approximation, the position of any individual molecule may be invariant within homogenous surroundings. But, by contrast, the molecular orientation usually undergoes stochastic changes between each absorption event, so that the observed absorption rate is calculated as an average of the rates over all orientations. In the account that follows in section 3 , the rotationally averaged rate calculations for one- and two-photon absorption, duly modified by an auxiliary beam, are derived and analyzed with special regard to the dependence on optical polarization. The paper ends with a conclusion section, including discussion of possible new dimensions in nonlinear optical spectroscopy arising from our mechanisms.

\section{LASER-MODIFIED ABSORPTION}

\subsection{Quantum electrodynamic framework}

In this quantum picture, the absorption rate is conventionally determined from the Fermi rule $\Gamma_{F I}=2 \pi \hbar^{-1} \rho_{F}\left|M_{F I}\right|^{2}$ using a standard perturbation expansion of the matrix element, $M_{F I}$ :

$$
\begin{aligned}
M_{F I}= & \left\langle F\left|H_{\mathrm{Int}}\right| I\right\rangle+\sum_{R} \frac{\left\langle F\left|H_{\mathrm{Int}}\right| R\right\rangle\left\langle R\left|H_{\mathrm{Int}}\right| I\right\rangle}{\left(E_{I}-E_{R}\right)}+\sum_{R, S} \frac{\left\langle F\left|H_{\mathrm{Int}}\right| S\right\rangle\left\langle S\left|H_{\mathrm{Int}}\right| R\right\rangle\left\langle R\left|H_{\mathrm{Int}}\right| I\right\rangle}{\left(E_{I}-E_{S}\right)\left(E_{I}-E_{R}\right)} \\
& +\sum_{R, S, T} \frac{\left\langle F\left|H_{\mathrm{Int}}\right| T\right\rangle\left\langle T\left|H_{\mathrm{Int}}\right| S\right\rangle\left\langle S\left|H_{\mathrm{Int}}\right| R\right\rangle\left\langle R\left|H_{\mathrm{Int}}\right| I\right\rangle}{\left(E_{I}-E_{T}\right)\left(E_{I}-E_{S}\right)\left(E_{I}-E_{R}\right)}+\ldots
\end{aligned}
$$

Equation (1) involves moving from an initial system (molecule plus all radiation fields) state $I$ to a final state $F$, the latter assigned to a continuum with density $\rho_{F}$, via virtual intermediate states $R, S$ and $T$. Appearing within the matrix element, the interaction Hamiltonian operator, $H_{\text {Int }}$, is evaluated via a mode expansion of the electric displacement; the electronic response of the molecule to each interaction is approximated as an electric dipole. In the electric dipole (E1) approximation, higher-order transitions are described using $\mathrm{E} 1^{2}, \mathrm{E} 1^{3}$, etc. represented by molecular response tensors $\alpha, \beta$, $\chi$, which are duly constructed from combinations of transition dipole moments $\mu$. In passing we note that the dipole coupling approximation is itself only fully defensible in a quantum electrodynamical framework, as Woolley has shown. ${ }^{5}$

\subsection{Description of the one-photon absorption process}

Single-photon absorption, resulting from the application of a resonant laser beam, generally involves a matrix element that is defined by first-order time-dependent perturbation theory. Commonly, where throughput electromagnetic radiation (independent of the absorbing beam) is not present, higher order terms are insignificant and they essentially denote self-energy corrections. However, on subjecting the system to an off-resonant (auxiliary) laser beam specifically a stimulus with an optical frequency at which the molecule is transparent - higher-order terms do indeed become significant. Under such conditions, there is no net absorption or stimulated emission of the off-resonant beam, but elastic forward-scattering events occur - photons are annihilated and created into the same radiation state (which thus emerges unchanged). These events engage though nonlinear coupling with one-photon absorption (Figure 1), the net effect being to modify the transition dipole moment corresponding to light absorption; this mechanism entails three molecule-photon interactions, i.e. a third order perturbation. 


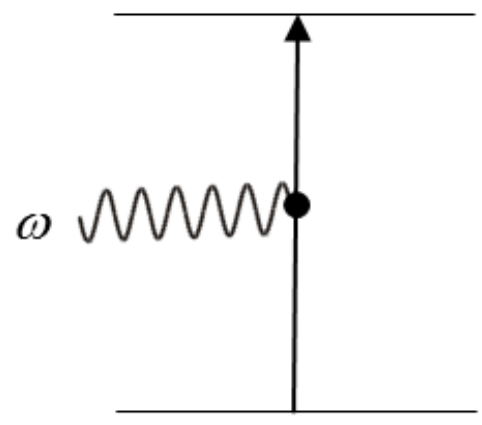

(a)

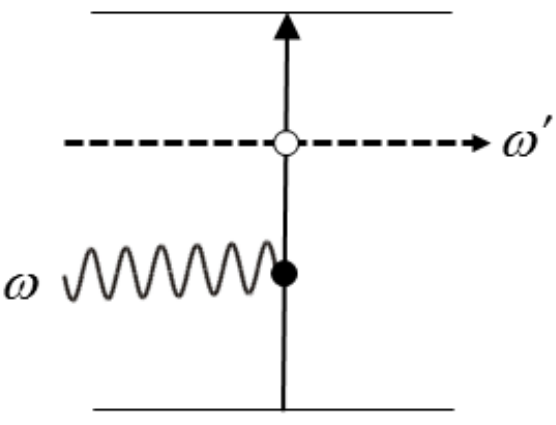

(b)

Figure 1. Energy level representation for; $(a)$ the linear interaction, $(b)$ the nonlinear interactions of laser-modified one-photon absorption. The wavy line denotes an incoming resonant photon $(\omega)$ and the vertical arrow is a transition due to the resulting light absorption. The nonlinear case also involves the stimulus laser beam $\left(\omega^{\prime}\right)$ shown by the horizontal dashed arrow; the black and open dots symbolize one- and two-photon interactions, respectively.

As a result of the quadratic (nonlinear) dependence of absorption rates, the rate equation for laser-modified one-photon $(1 \phi)$ absorption not only incorporates terms corresponding to first- and third-order perturbations, but also a cross term relating to a mixture of these perturbations - the latter term representing quantum interference. Explicitly, excluding media effects, the net rate for laser-modified one-photon absorption is determined as;

$$
\Gamma_{1 \phi}=\left(\frac{\pi \rho I}{c \hbar \varepsilon_{0}}\right) e_{i} \bar{e}_{j}\left[\mu_{i}^{(0) \alpha 0} \bar{\mu}_{j}^{(0) \alpha 0}+\left(I^{\prime} / c \varepsilon_{0}\right) \mu_{i}^{(0) \alpha 0} \bar{\mu}_{j}^{(2) \alpha 0}\left(\omega^{\prime}\right)+\left(I^{\prime 2} / 4 c^{2} \varepsilon_{0}^{2}\right) \mu_{i}^{(2) \alpha 0}\left(\omega^{\prime}\right) \bar{\mu}_{j}^{(2) \alpha 0}\left(\omega^{\prime}\right)\right],
$$

where $I$ is the irradiance of the absorbing (resonant) beam, $I^{\prime}$ is the throughput laser irradiance, and the overbar on a polarization component allows for the possibility of complex (such as circular) polarization. Equation (1) is interpreted as one-photon absorption associated with an effective excitation dipole whose zeroth order contribution (independent of the auxiliary beam) is a transition dipole moment between the ground, 0 , and excited, $\alpha$, state: $\boldsymbol{\mu}^{(0) \alpha 0}$. The contribution quadratic in the electric field of the auxiliary beam is $\mu_{i}^{(2) \alpha 0}\left(\omega^{\prime}\right) \equiv e_{j}^{\prime} e_{k}^{\prime} \beta_{i j k}^{\alpha 0}\left(\omega^{\prime}\right)$, where $\mathbf{e}^{\prime}$ is the polarization of the auxiliary beam and $\beta_{i j k}^{\alpha 0}\left(\omega^{\prime}\right)$ is the relevant transition hyperpolarizability. The former represents direct resonant absorption, and the latter a nonlinear correction term that couples with the off-resonant radiation of frequency $\omega^{\prime}$ (corresponding to elastic forward-scattering). The auxiliary beam thus effects a modification of the excitation dipole moment through $\boldsymbol{\mu}^{(2) \alpha 0}\left(\omega^{\prime}\right)$. It is the second term (linear in $\left.I^{\prime}\right)$, signifying a quantum interference of these two amplitudes, that represents the leading correction.

\subsection{Description of the two-photon absorption process}

Similar principles apply when one considers the effect of an auxiliary beam on two-photon absorption, where the fundamental process is considered a second-order perturbation. Irradiating the system with an off-resonant (auxiliary) beam results in a process entailing four molecule-photon interactions, namely a fourth-order perturbation; the outcome is that the two-photon rate of absorption may be affected. In detail, laser-modified two-photon absorption entails two identical interactions from the input resonant beam, along with the previously described elastic forward-scattering of the auxiliary beam. The rate expression for laser-modified two-photon absorption is; 


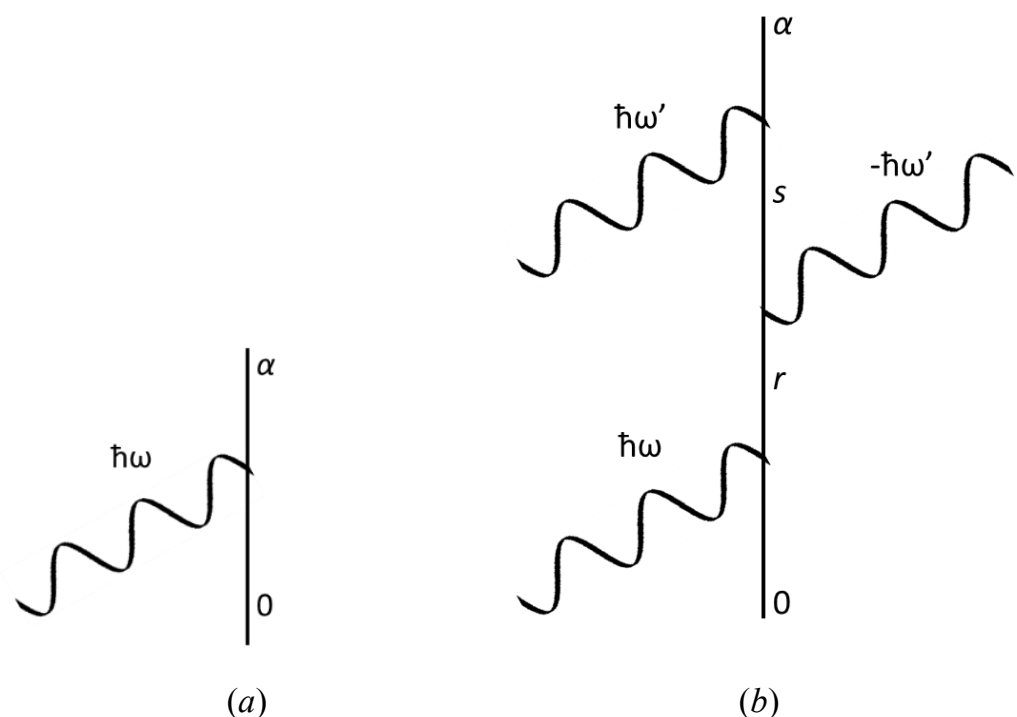

Figure 2. Feynman diagrams for: (a) elementary one-photon absorption; (b) one-photon absorption engaging with forward scattering of a passive beam (showing one of six time orderings).

$$
\Gamma_{2 \phi}=\left(\frac{\pi \rho I^{2}}{8 \hbar c^{2} \varepsilon_{0}^{2}}\right) e_{i} e_{j} \bar{e}_{k} \bar{e}_{l}\left[\alpha_{i j}^{(0) \alpha 0} \bar{\alpha}_{k l}^{(0) \alpha 0}+\left(I^{\prime} / c \varepsilon_{0}\right) \alpha_{i j}^{(0) \alpha 0} \bar{\alpha}_{k l}^{(2) \alpha 0}\left(\omega^{\prime}\right)+\left(I^{\prime 2} / 4 c^{2} \varepsilon_{0}^{2}\right) \alpha_{i j}^{(2) \alpha 0}\left(\omega^{\prime}\right) \bar{\alpha}_{k l}^{(2) \alpha 0}\left(\omega^{\prime}\right)\right] \text {. }
$$

Here, the transition polarizability $\alpha_{i j}^{(0) \alpha 0} \equiv \alpha_{i j}^{\alpha 0}$ denotes a zeroth order (auxiliary beam independent) contribution, and $\alpha_{i j}^{(2) \alpha 0} \equiv \boldsymbol{e}_{k}^{\prime} \bar{e}_{l}^{\prime} \chi_{i j k l}^{\alpha 0}\left(\omega^{\prime}\right)$ corresponds to a contribution quadratic in the electric field of the off-resonant beam; $\chi_{i j k l}^{\alpha 0}\left(\omega^{\prime}\right)$ is a second-order hyperpolarizability. The first term in equation (2) represents two-photon absorption, independent of the auxiliary beam. The third term signifies absorption in the presence of the elastically forward scattered off-resonant beam. Again it is the second, quantum interference term (linear in $I^{\prime}$ ) that represents the leading correction.

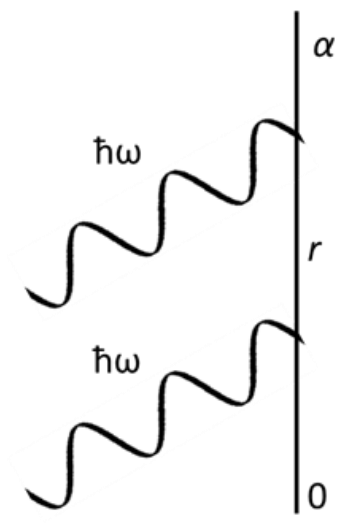

(a)

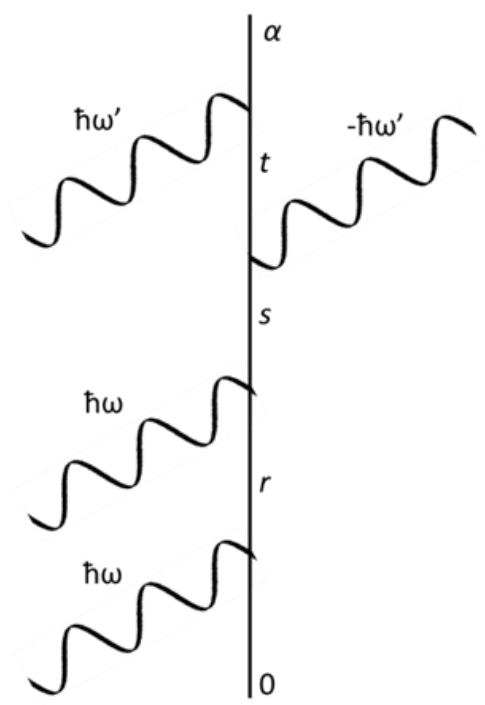

(b)

Figure 2. Feynman diagrams for: $(a)$ two-photon absorption; $(b)$ two-photon absorption engaging with forward scattering of a passive beam (showing one of 24 time orderings). 


\subsection{Population of metastable states}

It is also interesting that, on application of the off-resonant beam, electronic transitions that are normally forbidden may become active. Although the energy of either one or two photons within the resonant beam may match the difference between the energy levels of two electronic states (the lower of which is populated), absorption may yet not occur due to over-riding symmetry constraints. Such higher-energy (generally metastable) states may, nevertheless, be populated due to the higher-order processes since different selection rules apply for any such case. Namely, electronic states that are inaccessible to one and two-photon absorption may become available upon throughput of the off-resonant beam since, for example, a one-photon process has dissimilar selection rules compared to the three molecule-photon events that arise on application of the beam. Particular interest attaches to the fact that fluorescence emission from these same states will also be forbidden. Thus, once the stimulus beam has left the system, sample molecules may remain in states that are unusually long-lived. ${ }^{6}$ We expect that this prospect has potential energy trapping and photochemistry applications.

\section{AN EXPERIMENTAL SYSTEM: MOLECULES IN SOLUTION}

\subsection{Quantum description of medium-modified absorption}

The core of this paper examines a realistic experimental system, which involves individual molecules in solution. In this sub-section the quantum description of absorption, and the corresponding equations, is discussed. For the following purposes, however, some additional development of theory is required, to account for the medium effects on the optical interactions. It is the transverse electric displacement of the molecule, $\mathbf{d}^{\perp}$, that is directly modified by the optical properties of the medium. In solid media, a polariton formulation is appropriate; however, for the present application of fluidic surroundings we can regard the necessary modification as an electronic 'dressing' of the electromagnetic fields associated with each photon, cast in terms of the refractive index $n$. The relevant formulation can then be adapted from the condensed phase descriptions developed by Milonni and Juzeliūnas, ${ }^{7}$ and is given as follows;

$$
\mathbf{d}^{\perp}=i\left(\frac{\hbar \varepsilon_{0} v_{g} \omega}{2 c V}\right)^{1 / 2}\left(\frac{n_{(\omega)}^{2}+2}{3 n_{(\omega)}^{1 / 2}}\right)\left[\mathbf{e}_{(\eta, \mathbf{k})} a_{(\eta, \mathbf{k})}-\overline{\mathbf{e}}_{(\eta, \mathbf{k})} a_{(\eta, \mathbf{k})}^{\dagger}\right]
$$

It is unnecessary to include position-dependent phase factors, since this application relates to processes occurring in their entirety at a single position. Photon annihilation and creation operators $a_{(\eta, \mathbf{k})}$ and $a_{(\eta, \mathbf{k})}^{\dagger}$ operate on Fock number states.

Our most generalized model focuses upon a single molecule in solution. The rate results of the following sub-sections describe the auxiliary-beam-modified absorption behavior of a chromophore that is fixed in a static arrangement, yet is subject to the medium effects of refraction and dispersion. Before accounting for the effects of rotation in situ, we can recognize that the solution environment exerts influence through a certain refractive index $n$. The refractive index $n$ is defined for each radiation mode, with the group velocity $v_{g}$ defined accordingly: $:^{7,8}$

$$
v_{g} \equiv \frac{\partial \omega}{\partial k}=\frac{c}{n_{(\omega)}}
$$

\subsection{One-photon absorption}

The relevant terms for the case of one-photon absorption are the first- and third-order terms from equation (2), as illustrated in the Feynman diagram representation by Fig. 1. These are the leading terms, since even orders vanish for this interaction. In the following, the absorbed photon is distinguished from the auxiliary beam light by the latter's variables being marked with a prime: e.g. the condition that the auxiliary beam is off-resonance with the absorption is specified as $\hbar \omega^{\prime}<E_{\alpha 0}^{M}$. 
The result of evaluating the full set of contributions generated by the state summation is a tensor describing the molecule's third-order response to the microscopic electric fields. This is one form of the molecule's hyperpolarizability of the $\alpha \leftarrow 0$ transition, conventionally labelled $\beta_{i j k}^{\alpha 0}$. The Fermi rule then leads to the following result for the observable one-photon absorption rate:

$$
\begin{aligned}
\Gamma_{1 \phi}=\frac{\pi \rho_{F}}{\hbar}\left[\frac{I}{c \varepsilon_{0}}\right]\left(\frac{n^{2}+2}{3 n}\right)^{2} e_{i} \bar{e}_{j}\left\{\mu_{i}^{\alpha 0} \bar{\mu}_{j}^{\alpha 0}\right. \\
+\left[\frac{I^{\prime}}{c \varepsilon_{0}}\right]\left(\frac{n^{\prime 2}+2}{3 n^{\prime}}\right)^{2} \vec{e}_{k}^{\prime} e_{l}^{\prime} \operatorname{Re}\left(\mu_{i}^{\alpha 0} \bar{\beta}_{j k l}^{\alpha 0}\right) \\
\left.+\frac{1}{4}\left[\frac{I^{\prime}}{c \varepsilon_{0}}\right]^{2}\left(\frac{n^{\prime 2}+2}{3 n^{\prime}}\right)^{4} e_{k}^{\prime} \vec{e}_{l} \vec{e}_{m} e_{n}^{\prime} \beta_{i k l}^{\alpha 0} \bar{\beta}_{j m n}^{\alpha 0}\right\}
\end{aligned}
$$

The results are cast directly in terms of beam irradiance, through the relation $I \equiv \hbar c q \omega / V$, where $q$ is the number of photons in the quantization volume $V$, with a similar expression for the irradiance $I$ ' of the auxiliary beam - assuming sufficient intensity that $\left(q^{\prime}+1\right) / q^{\prime} \approx 1$. Here and below, use is made of the convention of summation over repeated vector or tensor component indices. The first term thus entails scalar products between the transition moment and polarization vectors; subsequent terms become more complicated through the involvement of tensor contractions involving the hyperpolarizability.

\subsection{Two-photon absorption}

For two-photon absorption, the second- and fourth-order terms from equation (2), as illustrated in the Feynman diagram representation by Fig. 2, dominate the matrix element. Odd orders vanish for this transition comprising two or four interaction events.

Due to the indistinguishability of the two absorbed photons, there is only one unique time-ordering for the isolated second-order process. This is equivalent to two one-photon absorption events from different beams, each with photon density of $q / 2 \mathrm{~V}$, at the limit of equal wavelength and parallel polarization. Under the Fermi rule, the above elements combine to give:

$$
\begin{aligned}
\Gamma_{2 \phi}=\frac{\pi \rho_{F}}{2 \hbar}\left[\frac{I}{c \varepsilon_{0}}\right]^{2}\left(\frac{n^{2}+2}{3 n}\right)^{4} e_{i} e_{j} \bar{e}_{k} \bar{e}_{l}\left\{\alpha_{i j}^{\alpha 0} \bar{\alpha}_{k l}^{\alpha 0}\right. & \\
& +\left[\frac{I^{\prime}}{c \varepsilon_{0}}\right]\left(\frac{n^{\prime 2}+2}{3 n^{\prime}}\right)^{2} \vec{e}_{m}^{\prime} e_{n}^{\prime} \operatorname{Re}\left(\alpha_{i j}^{\alpha 0} \bar{\chi}_{k l m n}^{\alpha 0}\right) \\
& \left.+\frac{1}{4}\left[\frac{I^{\prime}}{c \varepsilon_{0}}\right]^{2}\left(\frac{n^{\prime 2}+2}{3 n^{\prime}}\right)^{4} e_{m}^{\prime} \vec{e}_{n}^{\prime} \vec{e}_{o}^{\prime} e_{p}^{\prime} \chi_{i j m n}^{\alpha 0} \bar{\chi}_{k l o p}^{\alpha 0}\right\}
\end{aligned}
$$

The above expression applies when the absorbed light is a high-occupation number Fock (number) state, where it is reasonable to evaluate $(q-1) / q \approx 1$. More generally, one should introduce a pre-factor $g^{(2)}$, signifying the degree of second order coherence, ${ }^{9}$ containing radiation state information for the beam.

The quantum amplitude for a generic interaction between a single fixed molecule and $N$ photons is here expressed as a tensor product of the $N$ polarization vectors and the $N^{\text {th }}$-order molecular response tensor, with the $N$ wavevectors as arguments (the sign of these arguments indicating which photons are absorbed and which are emitted). These tensor contractions involve the Cartesian components of the molecular response tensors. 


\section{ROTATIONAL AVERAGE}

\subsection{Outline of the Method}

Each of the rate terms expresses an interaction between a molecular response tensor of even order and the relevant polarization unit vectors. The sources of the beams $I$ and $I^{\prime}$ are fixed in space, under laboratory control. Then the orientations of the fields specified by polarization vectors $\boldsymbol{e}$ and $\boldsymbol{e}$ ' define a space-fixed frame, such that each of the indices $i, j, k, l$ belongs to the corresponding space-fixed set $\{x, y, z\}$. Thus the right-hand factors of each rate term are components of the relevant molecular response tensors with respect to that frame.

A molecule's intrinsic electronic geometry (described by the molecular response tensors) is tied to the physical orientation of the molecule in space. The Cartesian components are invariant only if the molecule's orientation is fixed with respect to the laboratory frame. For a molecule able to reorient freely, the components of the tensors in their natural frame are invariant with respect to the molecule's orientation. So the tensors may be expressed in terms of a moleculefixed frame with the inclusion of a product of cosines relating the unit vectors. ${ }^{10,11}$

$$
T_{i j k l}=l_{i \lambda} l_{j \gamma} l_{k \varepsilon} l_{l \zeta} T_{\lambda \gamma \varepsilon \zeta}
$$

The indices $\lambda, \gamma, \epsilon, \zeta$ belong to the set of unit vectors that are the natural orthogonal triad for the molecule. The cosine product is now the only factor that varies as a function of the molecule's orientation. Explicitly, since rotations in the solution phase are not quantized, a rotational average is effected as a continuous integration over the three Euler angles relating the molecule-fixed frame to the space-fixed frame. Under such a rotational average the product of cosines $l_{i \lambda} l_{j \gamma} l_{k \varepsilon} l_{l \zeta}$ yields a sum of several Kronecker deltas relating the Latin and Greek indices, ${ }^{12}$ so the rotationally-averaged rate results are cast in terms of natural invariants of the tensor inner products (corresponding to particular symmetries). These scalar quantities signify the propensity of each molecule, in the course of a specific transition, to undergo the necessary charge redistribution consistent with the molecular geometry. ${ }^{13}$ In the results given below, $C_{v}^{u \phi}$ is the tensor contraction appearing in the right of the $v^{\text {th }}$ line of the $u$-photon absorption rate equation in section 3 . Chevron brackets \langle\rangle indicate operation of the rotational average.

\subsection{Effect on absorption rate results}

To address the simplest cases, we consider the extreme geometries of $\mathbf{e} \| \mathbf{e}^{\prime}$ (the polarization vectors of the absorbed and auxiliary beams are parallel) and $\mathbf{e} \perp \mathbf{e}^{\prime}$ (they are perpendicular). The directions of the Poynting vectors are not relevant, so any directions of beam incidence are allowed.

With parallel polarizations, each rotationally-averaged rate term becomes a weighted sum of all possible natural invariants.

$$
\begin{gathered}
\left\langle\mathrm{C}_{1}^{1 \phi}\right\rangle^{\mathrm{e} \| \mathrm{e}^{\prime}}=\frac{1}{3}\left(\mu^{\alpha 0} \cdot \bar{\mu}^{\alpha 0}\right) \\
\left\langle\mathrm{C}_{2}^{1 \phi}\right\rangle^{\mathrm{e} \| \mathrm{e}^{\prime}}=\frac{1}{15}\left[\mu_{\lambda}^{\alpha 0} \bar{\beta}_{\lambda \zeta \zeta}^{\alpha 0}+2 \mu_{\lambda}^{\alpha 0} \bar{\beta}_{\zeta \lambda \zeta}^{\alpha 0}\right] \\
\left\langle\mathrm{C}_{3}^{1 \phi}\right\rangle^{\mathrm{e} \| \mathrm{e}^{\prime}}=\frac{1}{105}\left(2 \beta_{\lambda \lambda \gamma}^{\alpha 0} \bar{\beta}_{\gamma \zeta \zeta}^{\alpha 0}+2 \beta_{\lambda \lambda \gamma}^{\alpha 0} \bar{\beta}_{\zeta \zeta \gamma}^{\alpha 0}+2 \beta_{\lambda \lambda \zeta}^{\alpha 0} \bar{\beta}_{\gamma \zeta \gamma}^{\alpha 0}+2 \beta_{\lambda \zeta \gamma}^{\alpha 0} \bar{\beta}_{\lambda \zeta \gamma}^{\alpha 0}\right. \\
\left.+2 \beta_{\lambda \zeta \zeta}^{\alpha 0} \bar{\beta}_{\gamma \zeta \gamma}^{\alpha 0}+2 \beta_{\lambda \zeta \gamma}^{\alpha 0} \bar{\beta}_{\zeta \lambda \gamma}^{\alpha 0}+2 \beta_{\lambda \zeta \gamma}^{\alpha 0} \bar{\beta}_{\gamma \lambda \zeta}^{\alpha 0}+\beta_{\lambda \zeta \zeta}^{\alpha 0} \bar{\beta}_{\lambda \gamma \gamma}^{\alpha 0}\right)
\end{gathered}
$$

These results are the fluid phase evaluations of the tensor components at the right end of each line of equation (6). 


$$
\begin{aligned}
& \left\langle\mathrm{C}_{1}^{2 \phi}\right\rangle^{\mathrm{e} \| \mathrm{e}^{\prime}}=\frac{1}{15}\left(\alpha_{\lambda \lambda}^{\alpha 0} \bar{\alpha}_{\zeta \zeta}^{\alpha 0}+2 \alpha_{\lambda \zeta}^{\alpha 0} \bar{\alpha}_{\lambda \zeta}^{\alpha 0}\right) \\
& \left\langle\mathrm{C}_{2}^{2 \phi}\right\rangle^{\mathrm{el| \textrm {e } ^ { \prime }}}=\frac{1}{105}\left(\alpha_{\lambda \lambda}^{\alpha 0} \bar{\chi}_{\gamma \zeta \zeta \zeta}^{\alpha 0}+2 \alpha_{\lambda \lambda}^{\alpha 0} \bar{\chi}_{\zeta \gamma \zeta \gamma}^{\alpha 0}+2 \alpha_{\lambda \zeta}^{\alpha 0} \bar{\chi}_{\lambda \zeta \gamma}^{\alpha 0}+2 \alpha_{\lambda \zeta}^{\alpha 0} \bar{\chi}_{\gamma \lambda \lambda}^{\alpha 0}+8 \alpha_{\lambda \zeta}^{\alpha 0} \bar{\chi}_{\lambda \gamma \zeta \zeta}^{\alpha 0}\right) \\
& \left\langle\mathrm{C}_{3}^{2 \phi}\right\rangle^{\mathrm{e} \| \mathrm{e}^{\prime}}=\frac{1}{945}\left(\chi_{\lambda \lambda \zeta \zeta}^{\alpha 0} \bar{\chi}_{\gamma \gamma \varepsilon \varepsilon}^{\alpha 0}+2 \chi_{\lambda \lambda \zeta \varepsilon}^{\alpha 0} \bar{\chi}_{\gamma \gamma \zeta \varepsilon}^{\alpha 0}+2 \chi_{\lambda \lambda \gamma \zeta}^{\alpha 0} \bar{\chi}_{\gamma \zeta \varepsilon \varepsilon}^{\alpha 0}+2 \chi_{\lambda \lambda \varepsilon \varepsilon}^{\alpha 0} \bar{\chi}_{\gamma \zeta \gamma \zeta}^{\alpha 0}\right. \\
& +8 \chi_{\lambda \lambda \gamma \varepsilon}^{\alpha 0} \bar{\chi}_{\gamma \zeta \zeta \varepsilon}^{\alpha 0}+2 \chi_{\lambda \gamma \zeta \zeta}^{\alpha 0} \bar{\chi}_{\lambda \gamma \varepsilon \varepsilon}^{\alpha 0}+4 \chi_{\lambda \gamma \zeta \varepsilon}^{\alpha 0} \bar{\chi}_{\lambda \gamma \zeta \varepsilon}^{\alpha 0}+8 \chi_{\lambda \gamma \zeta \zeta}^{\alpha 0} \bar{\chi}_{\lambda \zeta \varepsilon \varepsilon}^{\alpha 0} \\
& +8 \chi_{\lambda \gamma \varepsilon \varepsilon}^{\alpha 0} \bar{\chi}_{\lambda \zeta \gamma \zeta}^{\alpha 0}+16 \chi_{\lambda \psi \gamma \varepsilon}^{\alpha 0} \bar{\chi}_{\lambda \zeta \zeta \varepsilon}^{\alpha 0}+16 \chi_{\lambda \gamma \zeta \varepsilon}^{\alpha 0} \bar{\chi}_{\lambda \zeta \zeta \varepsilon}^{\alpha 0}+2 \chi_{\lambda \gamma \lambda \gamma}^{\alpha 0} \bar{\chi}_{\zeta \zeta \varepsilon \varepsilon}^{\alpha 0} \\
& \left.+2 \chi_{\lambda \gamma \varepsilon \varepsilon}^{\alpha 0} \bar{\chi}_{\zeta \zeta \lambda \gamma}^{\alpha 0}+8 \chi_{\lambda \gamma \lambda \varepsilon}^{\alpha 0} \bar{\chi}_{\zeta \zeta \gamma \varepsilon}^{\alpha 0}+4 \chi_{\lambda \gamma \lambda \gamma}^{\alpha 0} \bar{\chi}_{\zeta \varepsilon \zeta \varepsilon}^{\alpha 0}+4 \chi_{\lambda \gamma \zeta \varepsilon}^{\alpha 0} \bar{\chi}_{\zeta \varepsilon \lambda \gamma}^{\alpha 0}+16 \chi_{\lambda \gamma \lambda \zeta}^{\alpha 0} \bar{\chi}_{\zeta \varepsilon \gamma \varepsilon}^{\alpha 0}\right)
\end{aligned}
$$

These results likewise implement the tensor contractions in equation (7).

With perpendicular polarizations, not all of the possible natural invariants are allowed, as some involve implicit scalar products of the two beam polarizations. For one-photon absorption, the contractions in equation (6) become:

$$
\begin{gathered}
\left\langle\mathrm{C}_{1}^{1 \phi}\right\rangle^{\mathbf{e} \mathbf{e}^{\prime}}=\frac{1}{3}\left(\mu^{\alpha 0} \cdot \bar{\mu}^{\alpha 0}\right) \\
\left\langle\mathrm{C}_{2}^{1 \phi}\right\rangle^{\mathbf{e} \perp \mathbf{e}^{\prime}}=\frac{1}{15}\left[2 \mu_{\lambda}^{\alpha 0} \bar{\beta}_{\lambda \zeta \zeta}^{\alpha 0}-\mu_{\lambda}^{\alpha 0} \bar{\beta}_{\zeta \lambda \zeta}^{\alpha 0}\right] \\
\left\langle\mathrm{C}_{3}^{1 \phi}\right\rangle^{\mathbf{e} \perp \mathbf{e}^{\prime}}=\frac{1}{210}\left(5 \beta_{\lambda \lambda \gamma}^{\alpha 0} \bar{\beta}_{\gamma \zeta \zeta}^{\alpha 0}+12 \beta_{\lambda \lambda \gamma}^{\alpha 0} \bar{\beta}_{\zeta \zeta \gamma}^{\alpha 0}-2 \beta_{\lambda \lambda \zeta}^{\alpha 0} \bar{\beta}_{\gamma \zeta \gamma}^{\alpha 0}-2 \beta_{\lambda \zeta \gamma}^{\alpha 0} \bar{\beta}_{\lambda \zeta \zeta}^{\alpha 0}\right. \\
\left.-2 \beta_{\lambda \zeta \zeta}^{\alpha 0} \bar{\beta}_{\gamma \lambda \gamma}^{\alpha 0}-2 \beta_{\lambda \zeta \gamma}^{\alpha 0} \bar{\beta}_{\zeta \lambda \gamma}^{\alpha 0}-2 \beta_{\lambda \zeta \gamma}^{\alpha 0} \bar{\beta}_{\gamma \lambda \zeta}^{\alpha 0}-\beta_{\lambda \zeta \zeta}^{\alpha 0} \bar{\beta}_{\lambda \gamma \gamma}^{\alpha 0}\right)
\end{gathered}
$$

And for two-photon absorption, equation (7) is modified with:

$$
\begin{aligned}
& \left\langle\mathrm{C}_{1}^{2 \phi}\right\rangle^{\mathbf{e} \perp \mathbf{e}^{\prime}}=\frac{1}{15}\left(\alpha_{\lambda \lambda}^{\alpha 0} \bar{\alpha}_{\zeta \zeta}^{\alpha 0}+2 \alpha_{\lambda \zeta}^{\alpha 0} \bar{\alpha}_{\lambda \zeta}^{\alpha 0}\right) \\
& \left\langle\mathrm{C}_{2}^{2 \phi}\right\rangle^{\mathbf{e} \mathbf{e}^{\prime}}=\frac{1}{105}\left(3 \alpha_{\lambda \lambda}^{\alpha 0} \bar{\chi}_{\gamma \zeta \zeta \zeta}^{\alpha 0}-\alpha_{\lambda \lambda}^{\alpha 0} \bar{\chi}_{\gamma \zeta \zeta \zeta}^{\alpha 0}+6 \alpha_{\lambda \zeta}^{\alpha 0} \bar{\chi}_{\lambda \zeta, \gamma}^{\alpha 0}-\alpha_{\lambda \zeta}^{\alpha 0} \bar{\chi}_{\gamma \lambda \lambda \zeta}^{\alpha 0}-4 \alpha_{\lambda \zeta}^{\alpha 0} \bar{\chi}_{\lambda \gamma \zeta \gamma}^{\alpha 0}\right) \\
& \left\langle\mathrm{C}_{3}^{2 \phi}\right\rangle^{\mathbf{e} \mathrm{e}^{\prime}}=\frac{1}{630}\left(4 \chi_{\lambda \lambda \zeta \zeta}^{\alpha 0} \bar{\chi}_{\gamma \delta \varepsilon}^{\alpha 0}+8 \chi_{\lambda \lambda \zeta \varepsilon}^{\alpha 0} \bar{\chi}_{\gamma \gamma \zeta \varepsilon}^{\alpha 0}-\chi_{\lambda \lambda \gamma \zeta}^{\alpha 0} \bar{\chi}_{\gamma \zeta \varepsilon \varepsilon}^{\alpha 0}-\chi_{\lambda \lambda \varepsilon \varepsilon}^{\alpha 0} \bar{\chi}_{\gamma \zeta \zeta \zeta}^{\alpha 0}\right. \\
& -4 \chi_{\lambda \lambda \gamma \varepsilon}^{\alpha 0} \bar{\chi}_{\gamma \zeta \zeta \varepsilon}^{\alpha 0}+8 \chi_{\lambda \gamma \zeta \zeta}^{\alpha 0} \bar{\chi}_{\lambda \gamma \varepsilon \varepsilon}^{\alpha 0}+16 \chi_{\lambda \gamma \zeta \varepsilon}^{\alpha 0} \bar{\chi}_{\lambda \gamma \zeta \varepsilon}^{\alpha 0}-4 \chi_{\lambda \gamma \zeta \zeta}^{\alpha 0} \bar{\chi}_{\lambda \gamma \varepsilon \varepsilon}^{\alpha 0} \\
& -4 \chi_{\lambda \gamma \varepsilon \varepsilon}^{\alpha 0} \bar{\chi}_{\lambda \zeta \gamma \zeta}^{\alpha 0}-8 \chi_{\lambda \gamma \xi \varepsilon}^{\alpha 0} \bar{\chi}_{\lambda \zeta \zeta \varepsilon}^{\alpha 0}-8 \chi_{\lambda \gamma \zeta \varepsilon}^{\alpha 0} \bar{\chi}_{\lambda \zeta \zeta \varepsilon}^{\alpha 0}-\chi_{\lambda \gamma \lambda \gamma}^{\alpha 0} \bar{\chi}_{\zeta \zeta \zeta \varepsilon \varepsilon}^{\alpha 0} \\
& \left.-\chi_{\lambda \gamma \varepsilon \varepsilon}^{\alpha 0} \bar{\chi}_{\zeta \zeta \lambda \gamma}^{\alpha 0}-4 \chi_{\lambda \gamma \lambda \varepsilon}^{\alpha 0} \bar{\chi}_{\zeta \zeta \gamma \varepsilon}^{\alpha 0}+\chi_{\lambda \gamma \lambda \gamma}^{\alpha 0} \bar{\chi}_{\zeta \varepsilon \zeta \varepsilon}^{\alpha 0}+\chi_{\lambda \gamma \zeta \varepsilon}^{\alpha 0} \bar{\chi}_{\zeta \varepsilon \lambda \gamma}^{\alpha 0}+4 \chi_{\lambda \gamma \lambda \zeta}^{\alpha 0} \bar{\chi}_{\zeta \xi \gamma \varepsilon}^{\alpha 0}\right)
\end{aligned}
$$

The above equations, (9) - (20), are the core results for the analysis of auxiliary beam effects for single- and for twophoton absorption in solution. 


\section{DEPENDENCE ON THE POLARIZATION OF THE AUXILIARY BEAM}

The preceding section compares results for the extreme values of the angle $\cos ^{-1}\left(\mathbf{e} \cdot \mathbf{e}^{\prime}\right)$. The ratio of these two results quantifies the magnitude of the effect of this variable on each absorption rate, indicating the range of rate values that are experimentally attainable via manipulation of the auxiliary beam polarization $\boldsymbol{e}^{\prime}$. This ratio is similar in construction, and plays a similar role to, the depolarization ratio familiar in Raman spectroscopy. ${ }^{14}$

If the auxiliary beam is not involved in absorption (i.e. $I^{\prime}=0$ or $C_{3}$ is vanishing) then its polarization geometry is irrelevant and a ratio cannot be meaningfully assigned. For both one-and two-photon absorption, $C_{2}$ is derived from the product of $C_{1}$ and $C_{3}$, so if either of the latter are zero, $C_{2}$ must also vanish.

One case of particular interest is where one-photon absorption is only allowed through the involvement of the auxiliary beam, i.e. $\left\langle\mathrm{C}_{1}^{1 \phi}\right\rangle=0$, when it follows that $\Gamma_{1 \phi} \propto\left\langle\mathrm{C}_{3}^{1 \phi}\right\rangle$ and thus

$$
\frac{\Gamma_{1 \phi}^{\mathbf{e} \perp \mathbf{e}^{\prime}}}{\Gamma_{1 \phi}^{\mathbf{e} \| \mathbf{e}^{\prime}}}=\frac{\left\langle\mathrm{C}_{3}^{1 \phi}\right\rangle^{\mathbf{e} \perp \mathbf{e}^{\prime}}}{\left\langle\mathrm{C}_{3}^{1 \phi}\right\rangle^{\mathbf{e} \| \mathbf{e}^{\prime}}} .
$$

If all eight of the $\beta \beta$ natural invariant terms are equal, then this ratio evaluates as $1 / 5$. The ratio has its maximum value of 3 when $\beta_{\lambda \lambda \gamma}^{\alpha 0} \bar{\beta}_{\zeta \zeta \gamma}^{\alpha 0}$ dominates.

The same kind of observation applies to two-photon absorption: if $\left\langle\mathrm{C}_{1}^{2 \phi}\right\rangle=0$, then $\Gamma_{2 \phi} \propto\left\langle\mathrm{C}_{3}^{2 \phi}\right\rangle$ and thus

$$
\frac{\Gamma_{2 \phi}^{\mathbf{e} \perp \mathbf{e}^{\prime}}}{\Gamma_{2 \phi}^{\mathrm{e} \| \mathbf{e}^{\prime}}}=\frac{\left\langle\mathrm{C}_{3}^{2 \phi}\right\rangle^{\mathbf{e} \perp \mathbf{e}^{\prime}}}{\left\langle\mathrm{C}_{3}^{2 \phi}\right\rangle^{\mathbf{e} \| \mathbf{e}^{\prime}}}
$$

If all 17 of the $\chi \chi$ natural invariant terms are equal, then this ratio evaluates as $3 / 35$. Here, the depolarization ratio has its maximum value of 6 when $\chi_{\lambda \lambda \zeta \zeta}^{\alpha 0} \bar{\chi}_{\gamma \gamma \varepsilon \varepsilon}^{\alpha 0}, \chi_{\lambda \lambda \zeta \varepsilon}^{\alpha 0} \bar{\chi}_{\gamma \gamma \zeta \varepsilon}^{\alpha 0}, \chi_{\lambda \gamma \zeta \zeta}^{\alpha 0} \bar{\chi}_{\lambda \gamma \varepsilon \varepsilon}^{\alpha 0}$, or $\chi_{\lambda \gamma \zeta \varepsilon}^{\alpha 0} \bar{\chi}_{\lambda \gamma \zeta \varepsilon}^{\alpha 0}$ dominate. The detailed interpretation of these conditions is the subject of current ongoing analysis.

\section{DISCUSSION}

The dressed-photon formulation that has been applied in the above development, with refractive index $n$ explicitly engaged at the fundamental level, may be compared to alternative methods of correcting vacuum QED to account for medium effects. In a limiting case where $n=1$, our results reduce to a rotationally averaged form identical to the results in Ref. $^{15}$. The symmetry selection rules discussed there also apply here. Here, however, we have identified a straightforward depolarization measurement that has the capacity to assist interpretation of the symmetry rules. It will be interesting to explore how such applications can impact on the understanding of the relevant nonlinear interactions.

The higher-order molecular transitions are typically near-forbidden without an auxiliary beam involved in the interaction. Even with the very high-intensity primary stimulus that is feasible with current technology, selection rules demand a second beam, and the results of section 5 are representative of the important features that are relevant to this restriction.

Whilst it is possible that analytical applications will emerge, we consider the most important aspects of our work to be the identification and characterization of a fundamentally new kind of optical interaction. Clearly, there is scope for additional information to be gleaned by experimental manipulation of the greater number of controllable beam parameters. It is also interesting to reflect that, since the effects we have described produce no identifiable change in the emergent properties of the auxiliary beam, as it undergoes stimulated forward Rayleigh scattering, there may be scope 
for new kinds of quantum non-demolition measurement. Any demonstrable effect on the process of single- or twophoton emission from the pump radiation proves the presence of auxiliary beam photons without their overt annihilation.

\section{ACKNOWLEDGMENTS}

The authors are grateful to the Leverhulme Trust and the UK Engineering and Physical Sciences Research Council (EPSRC) for funding our research.

*david.andrews@physics.org

[1] Andrews, D. L., “Anomalous Absorption Of The Ultrafast-Laser Supercontinuum,” Phys. Rev. A 38, 5129-5139 (1988).

[2] Bradshaw, D. S. and Andrews, D. L., "Mechanism for Optical Enhancement and Suppression of Fluorescence,” J. Phys. Chem. A 113, 6537-6539 (2009).

[3] Bradshaw, D. S. and Andrews, D. L., "All-optical control of molecular fluorescence," Phys. Rev. A 81, 013424 (2010).

[4] Leeder, J. M., Bradshaw, D. S. and Andrews, D. L., "Laser-Controlled Fluorescence in Two-Level Systems," J. Phys. Chem. B 115, 5227-5233 (2011).

[5] Woolley, R. G., “Gauge Invariance and Multipole Moments,” Adv. Quantum Chem. 32, 167-180 (1998).

[6] Bradshaw, D. S. and Andrews, D. L., "Laser-modified one- and two-photon absorption: Expanding the scope of optical nonlinearity," Phys. Rev. A 88, 033807 (2013).

[7] Milonni, P. W., "Field Quantization And Radiative Processes In Dispersive Dielectric Media," J. Mod. Opt. 42, 1991-2004 (1995).

[8] Andrews, D. L. and Allcock, P., [Optical Harmonics in Molecular Systems], Wiley-VCH, Wienhiem (2002).

[9] Loudon, R., [The Quantum Theory of Light], Oxford Science Publications, Oxford (2000).

[10] McClain, W. M., "Polarization Dependence of Three-Photon Phenomena for Randomly Oriented Molecules," The Journal of Chemical Physics 57, 2264 (1972).

[11] Juzeliūnas, G., "Microscopic theory of quantization of radiation in molecular dielectrics: Normal-mode representation of operators for local and averaged (macroscopic) fields," Phys. Rev. A 53, 3543-3558 (1996).

[12] Craig, D. P. and Thirunamachandran, T., [Molecular Quantum Electrodynamics], Academic Press, London (1984).

[13] Andrews, D. L. and Ghoul, W. A., "Eighth rank isotropic tensors and rotational averages,” J. Phys. A: Math. Gen. 14, 1281-1290 (1981).

[14] Allemand, C. D., "Depolarization Ratio Measurements in Raman Spectrometry,” Appl. Spectrosc. 24, 348-353 (1970).

[15] Andrews, D. L. and Ford, J. S., "Resonance energy transfer: Influence of neighboring matter absorbing in the wavelength region of the acceptor," J. Chem. Phys. 139, 014107 (2013). 